\title{
Interferometric techniques in structural damage identification
}

\author{
H. Lopes ${ }^{\mathrm{a}}$, J. Ribeiro ${ }^{\mathrm{a}}$ and J.V. Araújo dos Santos ${ }^{\mathrm{b}, *}$ \\ ${ }^{a}$ ESTIG, Instituto Politécnico de Bragança, Campus de Sta. Apolónia, Bragança, Portugal \\ ${ }^{\mathrm{b}}$ IDMEC, Instituto Superior Técnico, Universidade Técnica de Lisboa, Lisboa, Portugal
}

\begin{abstract}
This paper describes several interferometric techniques and their applications in structural damage identification. With that objective in mind, damaged aluminum beams with clamped-free and free-free boundary conditions are analyzed. Different damages cases are inflicted by creating small cuts perpendicular to the beams longitudinal axis, being the damage, therefore, characterized by the dimensions of these cuts. The out-of-plane displacement field of modal response is measured with an electronic speckle pattern interferometric system. The static and dynamic rotation fields, defined as the spatial derivative of the displacement field, are measured with two different speckle shearography systems. Second and third order spatial derivatives of the displacement field, which are related to the bending moment and shear force, are computed by differentiation techniques. The cuts locations are determined by looking for maximum values and/or perturbations of damage indicators based on bending moments and shear forces. This method is validated by comparing its results with numerical ones, from which the most suitable interferometric technique is chosen.
\end{abstract}

Keywords: Damage identification, ESPI, speckle shearography, higher order spatial derivatives, beam

\section{Introduction}

The pursue of reliable structural damage identification methods has attracted the scientific and technical community for decades. Namely, in the last three decades, the characterization of the damage has been done around the study of delamination in composite materials, motivated by their growing use in the transportation industry and the absence of effective techniques for their detection. Among many methods developed over the years, those based on structural dynamic characteristics have been widely studied and applied [1]. These methods rely on the evidence that if the mass, stiffness and damping of a structure suffer changes due to damage, its dynamic response in the damaged state will differ from the response in the undamaged or original state. Therefore, by knowing these responses it is possible to detect, locate and even quantify the damage.

Although the proposed methodologies allow the full characterization of the structural damage by numerical simulations, they often fail when applied to experimental data. This problem is related to the fact that experimental techniques only allow the direct measurement of the structure displacement and rotation fields, which are affected by experimental noise. To overcome these limitations there are several approaches to this problem and different methodologies have been developed over the years.

The changes and perturbations in curvature fields are consistently referred in the literature as one of the most promising methodology for damage localization. This methodology was initially proposed by Pandey et al. [2]. In their method, the location of damage is based on the analysis of modal curvatures differences, obtained from the central finite difference of natural modes shapes. However, the use of sparse measurements of the modal displacement and the presence of experimental noise, which is propagated and amplified by the numerical differenti-

*Correctonding author: J.V. Araújo dos Santos, Instituto Superior Técnico, Universidade Técnica de Lisboa, Av. Rovisco Pais, 1049-001 Lisboa, Portugal. E-mail: viriato@ist.utl.pt. 
ation procedure, may lead to false locations of damages. New and improved methods, which are also based on the knowledge of curvature fields, have been proposed over the years [3-11].

Interferometric techniques are full field, non-contact and high resolution techniques that allow both static and dynamic measurements of a surface motion [12]. Nowadays, there are two main types of interferometric techniques: (1) electronic speckle pattern interferometry (ESPI), also known as TV-holography, and (2) digital speckle shearography or digital speckle pattern shearing interferometry. A comprehensive description of these techniques can be found in $[13,14]$. One of the first works in the classification of defects in a metal plate using a holographic interferometric technique was reported by Glunder and Lenz [15]. A fringe pattern of the component surface deformation is measured during the application of a load. The evaluation of structural damage relies on the recognition and classification of high or low fringe density in either the spatial or frequency domain. However, the automatic recognition of these patterns leads to fault indications of damage, due to the complexity of patterns within noisy images. The fringe density analysis becomes more difficult when dealing with complex geometry components containing inhomogeneities, i.e., sandwich panels. Damage identification methods based on fringe pattern classification have been improved by the application of neural networks techniques and the combination of experimental measurements and numerical simulations results [16-18]. The speckle shearography technique is a very promising technology to be used on an industrial environment. It allows the direct measurement of surface displacements derivatives. Being a very high resolution technique it is very robust to external perturbations and to rigid-body motions [19]. The inspection with a speckle shearography system of an aircraft sandwich panel, which was loaded with a flash light, allowed the localization of some of its internal damages [20]. In this case, the damage is revealed by the appearance of a concentric fringe pattern or by an alteration of their spacing, which result from localized deformations produced by the change in thermal conductivity. New developments have been made in the last few years with the combined use of different non-contact excitation sources [21].

The introduction of temporal or spatial phase modulation techniques for full evaluation of the phase maps of the interference pattern have allowed the increase in resolution of the measurements and the subsequent evaluation of the surface response field [12]. In these cases, the damage identification is performed based on local perturbations of the measured fields. Despite this improvement in the quality of the measurements, they are insufficient for the detection of small damages located away from the surface and also for the quantification of its severity in composites material. This means that a higher order derivative of the displacement field is required to fully characterize the damage, as referred earlier, e.g., the curvature field. Several approaches for the direct measurement of curvature fields, based on speckle shearography systems, were reported in recent years [22-24]. However, these approaches rely on systems that are not able to achieve curvature fields of good quality or the measurement procedure is too complex and difficult to implement.

Given the difficulties in the direct measurement of curvature fields, they are instead computed by numerical differentiation of the measured displacement or rotation fields. In the context of damage identification, Pandey et al. [2] applied a numerical differentiation of modal displacement fields using the central differences method. This technique shows to be ineffective, since it relies on sparse experimental noisy measurements. In order to cope with the propagation and amplification of experimental noise, more robust methods of numerical differentiation have been proposed [25-28].

The present analysis comprises static and modal measurements of out-of-plane displacement and rotation fields. A speckle shearography system with temporal phase modulation is used for the static measurement of the rotation field, while the modal measurement of the displacement and the rotation fields are obtained using ESPI and speckle shearography systems, with spatial phase modulation, which are designated as pulsed ESPI and pulsed speckle shearography to distinguish them from the static measurement system.

The measured fields obtained with these different systems are post-processed in order to compute other relevant fields. For instance, the static displacement field is obtained by numerical integration of the static rotation field. Similarly, the second and third spatial derivatives of the static rotation field, corresponding to the beam curvature and its derivative are determined by numerical differentiation. For the modal measurements, the beam rotation, curvature and its derivative are computed by numerical differentiation of the modal displacement field. Based on the second and third derivatives of the displacement fields and making use of the Euler-Bernoulli beam theory, the bending moment and the transverse force are determined. Finally, the damage identification is performed using the 
proposed displacement, rotation, bending moment and shear force damaged indicators.

\section{Methodology and results}

\subsection{Beam analysis}

\subsubsection{Characterization of damage}

In this study, the damages are inflicted by creating two small cuts perpendicular to the longitudinal axis of the beam using a small cutting saw and a small rasp. The clamped-free and free-free aluminum beams analyzed are $L=$ $260 \mathrm{~mm}$ long, $b=45 \mathrm{~mm}$ wide and $h=6 \mathrm{~mm}$ thick. In order to study the geometry of the damages, located at $l_{1}=$ $75 \mathrm{~mm}$ and $l_{2}=130 \mathrm{~mm}$ (Fig. 1), several cases are analyzed and their dimensions are shown in Table 1. The damage parameter $\delta_{k}$, also presented in this Table, relates the local stiffness decrease and is determined by:

$$
\delta_{k}=1-\frac{E \tilde{I}}{E I}=1-\frac{\tilde{I}}{I}=3\left(\frac{p_{k}}{h}\right)-6\left(\frac{p_{k}}{h}\right)^{2}+4\left(\frac{p_{k}}{h}\right)^{3}
$$

where $E$ is the Young's modulus and $I$ and $\tilde{I}$ are, respectively, the second moment of area of the undamaged and damaged transverse section.

Table 1

Damage cases, cuts dimensions [mm] and damage parameter

\begin{tabular}{lcccc}
\hline & Case 1 & Case 2 & Case 3 & Case 4 \\
\hline$p_{1}\left(\delta_{1}\right)$ & $0.4(0.175)$ & $0.65(0.26)$ & $0.65(0.26)$ & $0.65(0.26)$ \\
$c_{1}$ & 4 & 4 & 4 & 4 \\
\hline$P_{2}\left(\delta_{2}\right)$ & - & - & $0.4(0.175)$ & $0.75(0.29)$ \\
$c_{2}$ & & & 0.4 & 0.75 \\
\hline
\end{tabular}

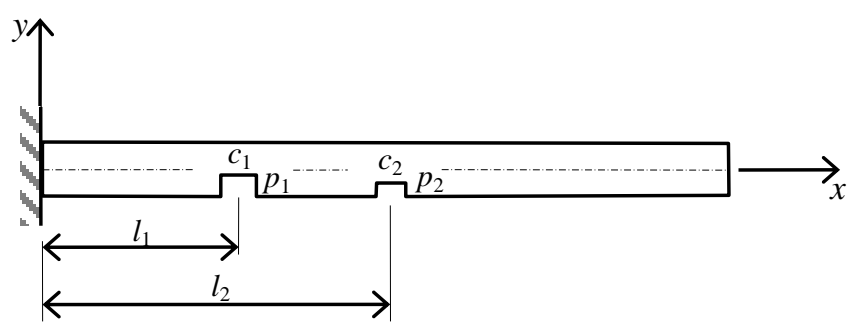

Fig. 1. Clamped-free aluminum beam with two cuts.

\subsubsection{Static response}

The static analysis of the clamped-free aluminum beam is based on the application of a $0.6 \mathrm{~N}$ force at the tip, using a Micro-Control ${ }^{\circledR}$ positioner. The static rotation field is measured using a speckle shear interferometric system with a temporal phase modulation technique. The angle of one of the Michelson interferometer mirrors is adjusted to provide an object plane shear around $13.5 \mathrm{~mm}$ along the longitudinal direction of the beam [12]. A Coherent ${ }^{\circledR}$ continuous laser with a $532 \mathrm{~nm}$ wave length is used to illuminate the measured surface. The registration of phase differences of two instants of time allows the comparison of an object distinct states and the measurement of the displacement field gradient. The system comprises the application of phase filtering and the unwrapping of the measured phase maps. 
The displacement field $y(x)$ is obtained by numerical integration of the rotation field $\theta(x)$. Similarly, the first and second spatial derivatives of the rotation field, corresponding to the beam curvature and its derivative, are determined by numerical differentiation. Based on the second and third derivatives of the displacement fields and making use of the Euler-Bernoulli beam theory, the bending moment $M(x)$ and the transverse force $V(x)$ are determined, as a function of $y(x)$ or of $\theta(x)$, respectively, by

$$
M(x)=E I \frac{d^{2} y(x)}{d x^{2}} \quad \text { and } \quad V(x)=E I \frac{d^{3} y(x)}{d x^{3}} \quad \text { or } \quad M(x)=E I \frac{d \theta(x)}{d x} \quad \text { and } \quad V(x)=E I \frac{d^{2} \theta(x)}{d x^{2}}
$$

Figure 2 presents the displacement field indicator (DFI), rotation field indicator (RFI), bending moment indicator (BMI) and shear force indicator (SFI), for the first and second damage cases described in Table 1. These indicators are defined, respectively, by:

$$
\begin{aligned}
& \operatorname{DFI}\left(x_{l}\right)=\left|\frac{\tilde{y}\left(x_{l}\right)-y\left(x_{l}\right)}{\max [y(x)]-\min [y(x)]}\right|, \quad \operatorname{RFI}\left(x_{l}\right)=\left|\frac{\tilde{\theta}\left(x_{l}\right)-\theta\left(x_{l}\right)}{\max [\theta(x)]-\min [\theta(x)]}\right| \\
& \operatorname{BMI}\left(x_{l}\right)=\left|\frac{\tilde{M}\left(x_{l}\right)-M\left(x_{l}\right)}{\max [M(x)]-\min [M(x)]}\right|, \quad S F I\left(x_{l}\right)=\left|\frac{\tilde{V}\left(x_{l}\right)-V\left(x_{l}\right)}{\max [V(x)]-\min [V(x)]}\right|
\end{aligned}
$$

where the tilde denotes the damaged beam quantities and $x_{l}$ is the coordinate.
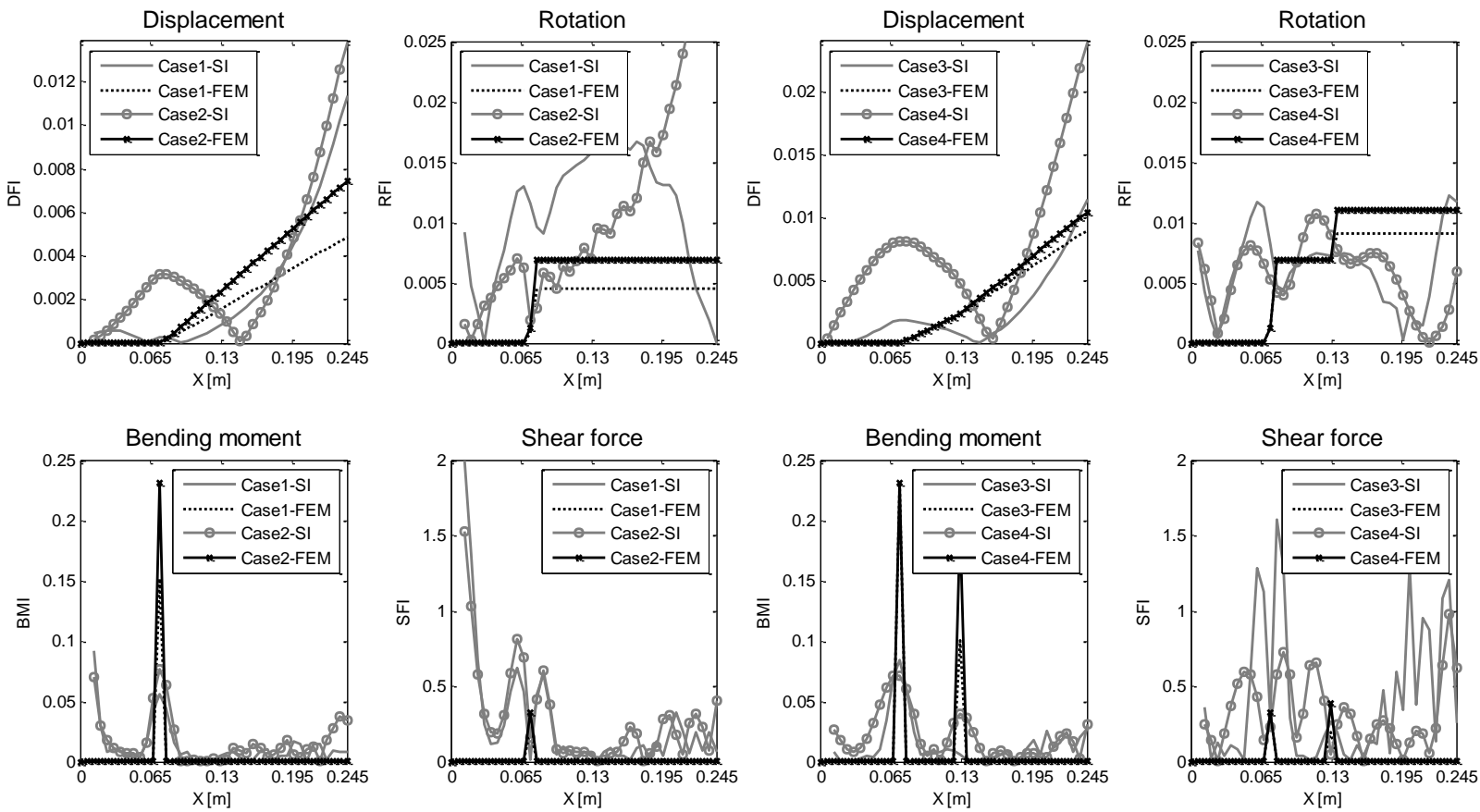

Fig. 2. DFI, RFI, BMI and SFI values for the four damage cases.

In order to validate the present method, both numerical data, obtained using the finite element method (FEM) and experimental data was used. From the comparative analysis, it is found that the experimental displacement fields (DFI), rotation fields (RFI) and shear force (SFI) indicators are globally distinct from the numerical ones. However, that is not the case of the bending moment indicator (BMI), which, with the exception of a region near the clamped end, presents a good agreement. Also, and more importantly, the maximum values of this indicator coincide with the 
cut position, allowing therefore its localization. Since in the second damage case the cut is larger, the BMI is also larger. There is an attenuation of the experimental value relatively to the one obtained through finite element analysis. In fact, smoothing averaging low-pass filters are applied to the rotating field in order to eliminate high frequency measurement noise [29]. An iterative and heuristic procedure was conducted in order to choose the optimal filter, which simultaneously preserves the signal components and eliminates high-frequency noise. However, this also leads to the suppression of high order terms of the signal that are crucial to the description of the high order derivatives. Nevertheless, one can compute the ratio between the maximum values of BMI for damage case 1 and 2 , which is 1.39 . This value is close to the one computed using the ratio of theoretical damage parameter in equation (1): $0.26 / 0.175=1.48$. It can also be seen in Fig. 2 that the SFI indicator is zero at the damage location, which is expected since the BMI is maximum.

The DFI, RFI, BMI and SFI values of the third and fourth damage cases are also shown in Fig. 2. For these damage cases, which consist in two cuts, the best damage localizations are achieved with the BMI. Indeed, the maximum values of this indicator are located at the cuts positions, although the amplitude are different from the ones obtained by the finite element method. This is similar to what is observed in the first and second damage cases. Also, for the third damage case, the second damage is not identified. All these problems are due to the application of low-pass filters to the experimental data. The relative quantification, which was possible in the first and second cases by relating the ratios between the maximum values of BMI is not achieved for the third and fourth damage cases. Among other factors, this is due to the difference of the cuts longitudinal dimensions $\left(c_{1}=4 \mathrm{~mm}\right.$ and $c_{2}=$ $0.4 \mathrm{~mm}$ or $c_{2}=0.75 \mathrm{~mm}$ ), leading to an attenuation of the damage parameter in the second cut. In other words, the damage influence of the second cut is mix up with the high frequency noise, being suppressed by applying low-pass filters in the differentiation procedure.

\subsubsection{Modal response}

The application of the present damage indicators, DFI, RFI, BMI, and SFI, to modal response measurements is described next. Natural frequencies are determined by classical experimental modal analysis procedures and the mode shapes are obtained using pulsed ESPI and pulsed speckle shearography systems. The main advantages of these techniques are the noncontact nature of the mode shape measurements, in opposition to the common modal analysis experimental techniques, such as performing the excitation using a hammer and the response measurement using an accelerometer.

The mode shapes are measured based on the identified natural frequencies and using the pulsed ESPI and pulsed speckle shearography systems. The ESPI system relies on the digital recording, using a CCD camera, of the phase difference of two wave fronts optical path. The ESPI system for dynamic measurements, also known as double pulse TV holography, uses holographic interferometry to compare two states of an object in a very short time interval. A commercial pulsed ESPI system (Ettemeyer $\mathrm{GmbHCo}^{\circledR}$ ) was used to measure the mode shapes displacement fields of the free-free beam. This system uses a LUMONICS ${ }^{\circledR}$ double pulse Ruby laser to generate a pair of impulses with $690 \mathrm{~nm}$ wave length, separated by $400 \mu \mathrm{s}$. The double exposure holograms are recorded by a $512 \times 512$ resolution CCD camera and afterwards treated with image processing techniques.

The beam was suspended with rubber bands and excited acoustically at each natural frequency using a loudspeaker. This is accomplished by using a Tektronix ${ }^{\circledR}$ model AFG320 signal generator. At each frequency, the signal amplitude was adjusted in order to fit the amplitude of the vibration within the range of the measurement system. This signal is also used to synchronize the laser trigger and the harmonic vibration of the beam. The primary phase map of each holographic recording is obtained by demodulation of the spatial carrier introduced in both holograms [12]. By subtracting them, the phase map of the surface motion is obtained. These phases maps are post-processed using dedicated image processing techniques to obtain the three dimensional representation of the measured field. In this procedure, the averaging filters are applied to the phase maps to remove the high frequency speckle noise and afterwards the Flynn phase unwrapping method is applied to remove the phase map discontinuities. A comprehensive description of these techniques can be found in $[12,30]$. One can define the displacement field of a mode shape as a vector of norm one by taking the amplitudes of the longitudinal axis at half width of the beam. An excellent agreement was obtained between the modal analysis, ESPI measurements and finite element analysis, allowing us to validate the ESPI technique. 

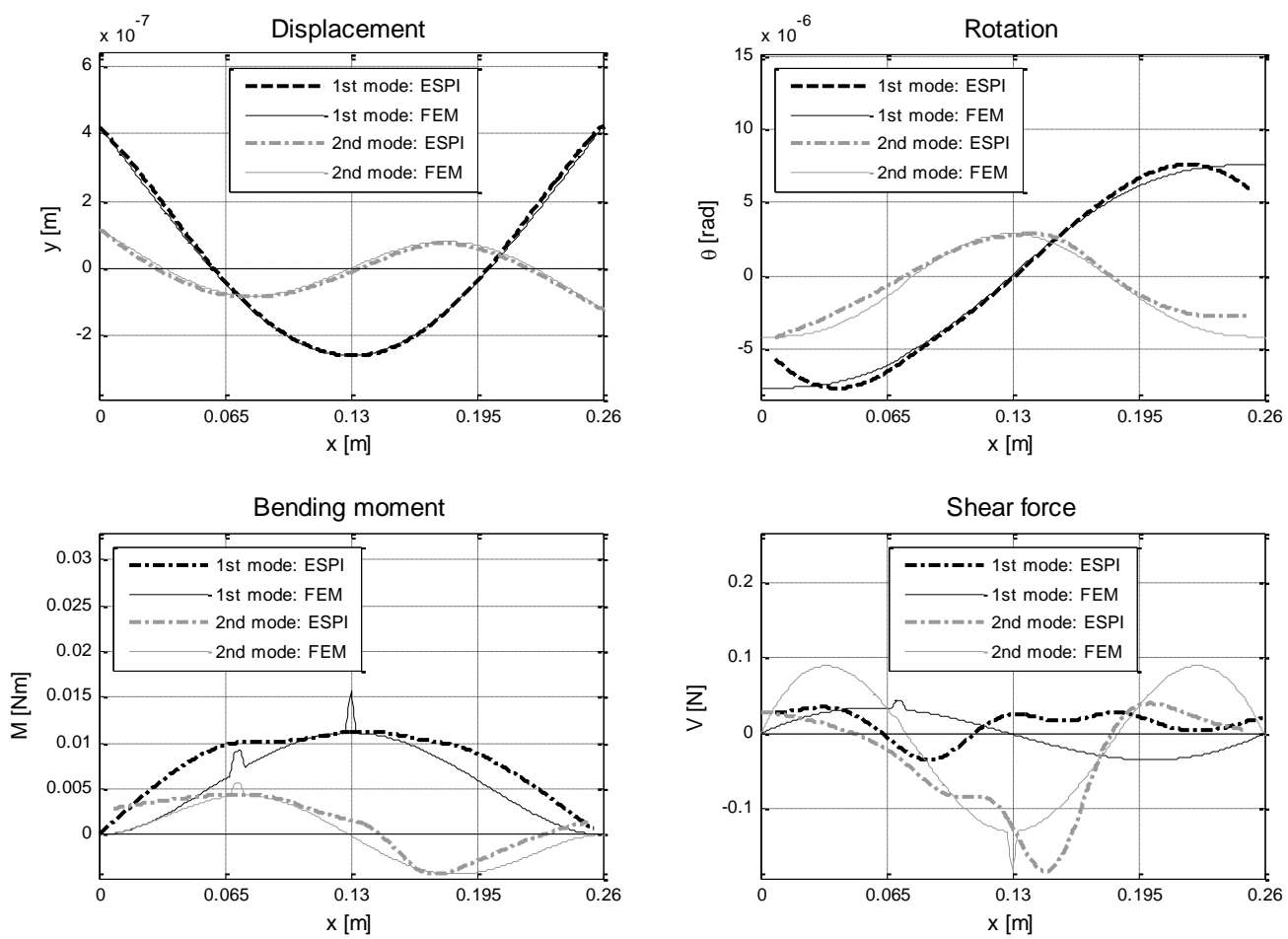

Fig. 3. Displacement and rotation fields, bending moments and shear force distributions of damaged beam using the pulsed ESPI system (ESPI) and the finite element method (FEM) for the fourth damage case.
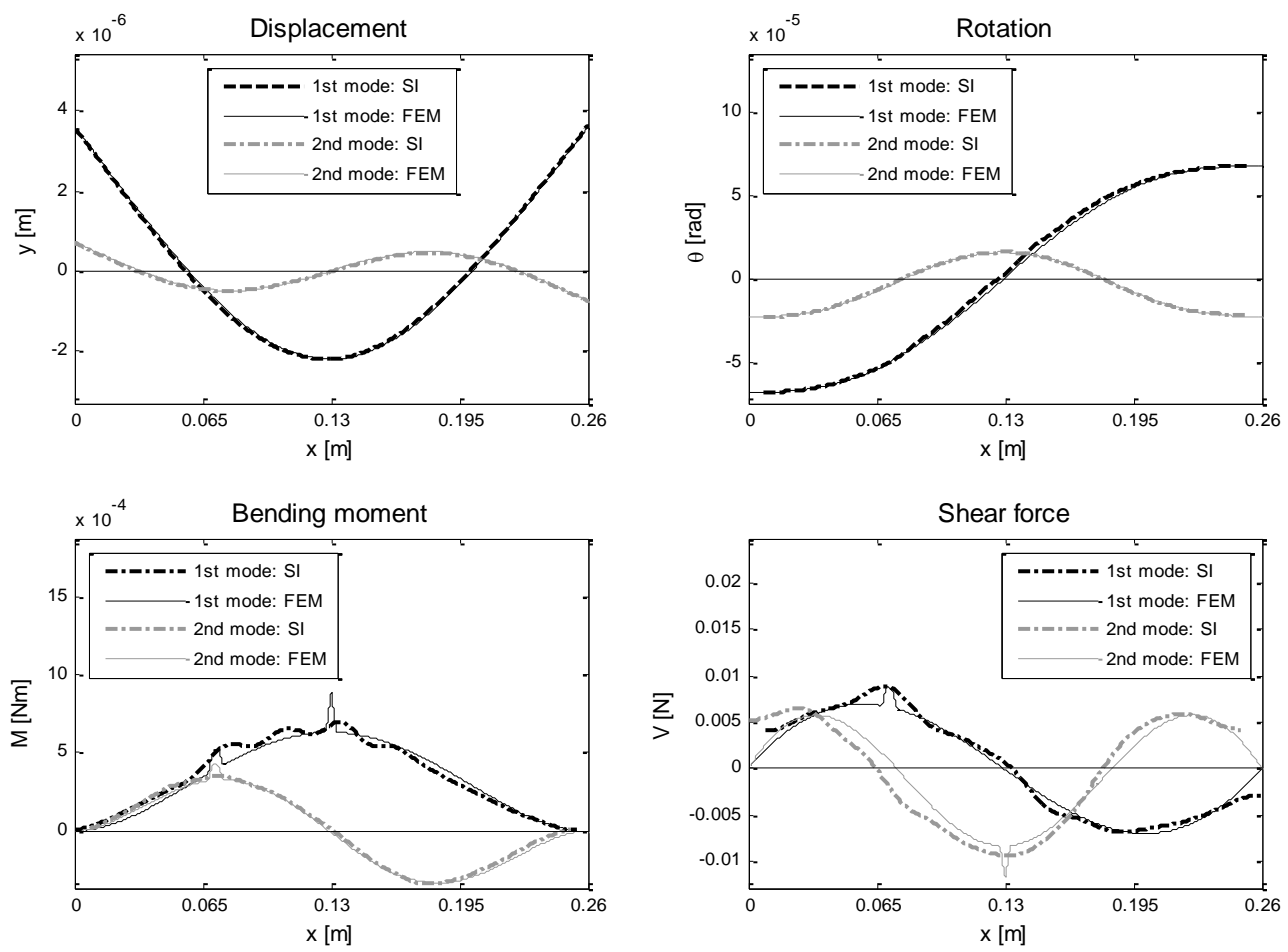

Fig. 4. Displacement and rotation fields, bending moments and shear force distributions of damaged beam using the pulsed shear interferometric system (SI) and the finite element method (FEM) for the fourth damage case. 
The pulsed speckle shearography system for the dynamic measurements is based on a Mach-Zehnder interferometer optical setup and uses a double pulsed laser to measure, in two consecutive instants, the displacement field gradient of the surface motion. This system also uses the LUMONICS ${ }^{\circledR}$ double pulse Ruby laser used in the ESPI measurements. Similarly to the ESPI system, a spatial carrier introduced in the primary fringes of each hologram leads to their phase distribution $[31,32]$. The interferogram of the rotation field, i.e. the displacement field gradient, is constructed based on these two holographic recordings

The rotation field measurements of the mode shapes were performed with the same experimental setup as the one used to measure the displacement field with the ESPI system. However, in order to frame the amplitude of rotations into the measurement range, the laser pulse was synchronized with the mode shape vibration amplitude. For that purpose, a trigger level was defined as described in [26]. This system is also insensitive to uniform displacements of the structure under study, therefore granting the confinement of rigid body motion. The rotation field is obtained by post-processing the interferogram phase maps, using the same techniques describe for the ESPI technique. The rotation fields obtained by the speckle shearography system and by finite elements show identical behavior, therefore validating the experimental measurements.

The procedure of damage identification based on the modal response is similar to that based on the static response. Indeed, we make use of the same indicators, i.e. the DFI, RFI, BMI, and SFI indicators, and the damage induced to the free-free aluminum beam is the same as the fourth damage case of the clamped-free aluminum beam presented in Table 1. The displacement and rotation fields are measured by the pulsed ESPI and pulsed speckle shearography system, respectively. The curvature and its derivative are obtained by numerically differentiating these fields, which are afterwards related to the bending and shear force using the Euler-Bernoulli beam theory (see Eq. (2)).

The displacement and rotation fields and the bending moments and shear distributions of the first two mode shapes of the damaged beam using the pulsed ESPI system and the finite element method are shown in Fig. 3. The rotation fields and the distributions of bending moment and shear force are computed by numerical differentiation of the displacement field. These same fields and distributions, obtained using the pulsed speckle shearography system, are presented in Fig. 4. Note that since this system measures the rotations, the displacement field is obtained by numerical integration, while the bending moment and shear force distributions are computed by differentiation. By comparing the data in Figs 3 and 4, we observe that the rotation fields obtained with the pulsed speckle shearography system are closer to those of the finite element method. Indeed, there are significant differences between the ESPI rotation fields and the finite element ones, namely at the edges of the beam. These differences become even larger in the bending moment and shear force distributions, which are computed by differentiating twice and thrice the displacement field, respectively. Since the bending moment and shear force distributions based on the pulsed speckle sherography system measurements are computed by differentiating the rotation field once and twice, respectively, the propagation of measurement noise and errors in this case is lower than the one observed in the ESPI measurements.

The changes produced by the damage are much larger in the bending moment and shear force than in the rotation and displacement fields, where almost no discontinuities and perturbations are visible. This is particularly visible in Fig. 4. However, the localization of damage is not completely clear, due to superposition of perturbations and noise in the measurement signal and discontinuities and perturbations due to damage. These signal perturbations and errors can be isolated by applying the damage indicators proposed and, therefore, an increase on damage localization sensitivity can be accomplished. Figure 5 shows the BMI and SFI values of the first and second mode shapes computed using the measurements taken with the pulsed ESPI system, the pulsed shear interferometric system and the results from a finite element analysis. As expected, a better damage localization is accomplished using the pulsed speckle sherography system and the BMI indicator. Indeed, by looking at the BMI distributions, one sees that the BMI computed with the pulsed shear measurements presents a similar trend to the BMI distribution computed based on the finite element method results. It also presents maximum values at the beam sections where the cuts are located. The SFI indicator is not able to locate the cuts, since, as stated before, the shear force is computed by differentiating twice the rotation field measured with the pulse speckle sherography system and differentiating thrice the displacement field measured with the ESPI system. These differentiations are based on the simultaneous application of signal differentiation and high frequency filtering techniques, as described previously. In this process, the signal high frequencies, needed to represent the perturbations due to damage and which are usually mixed up 
with high frequency measurement noise, are eliminated. The elimination of these perturbations is more significant in higher modes, being this the reason why the second mode presents a poor damage localization, when compared with the damage localization achieved with the first mode. It is also important to note that the damage localizations using the static measurements are better than the ones obtained using the modal measurements for the same reason, i.e., since the static rotation field is represented by low order terms in the wave number domain [26], the elimination of perturbations due to damage is much lower.
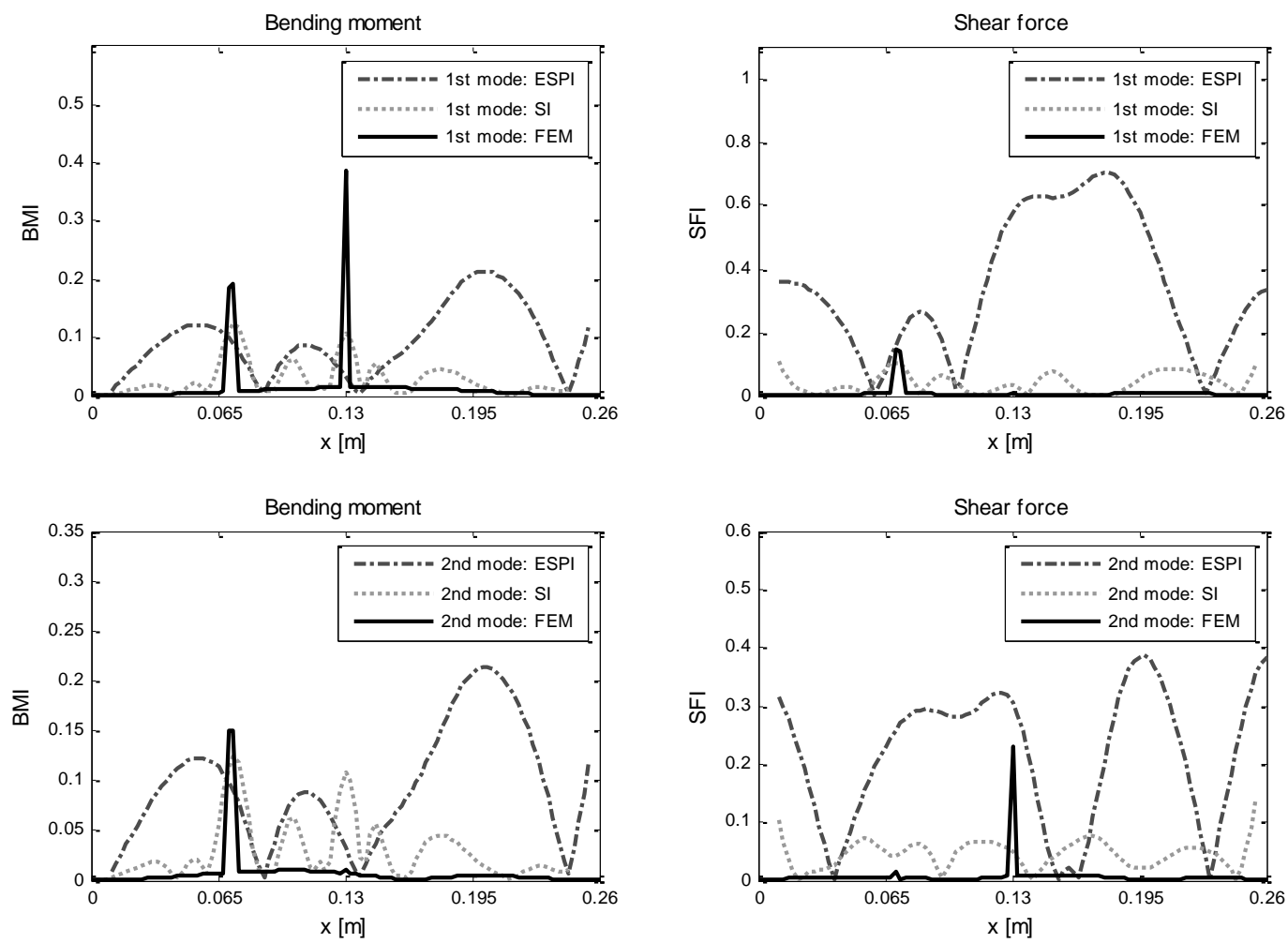

Fig. 5. BMI and SFI values of the first and second mode shapes using the pulsed ESPI, pulsed shear (SI) interferometric systems and the finite element method (FEM) for the fourth damage case.

\section{Conclusions}

Several interferometric techniques and their applications to damage localization of cuts in beams were described in this paper. The measurements of the displacement and rotation fields using ESPI and speckle shearography systems, respectively, and subsequent application of a numerical differentiation method, allowed us to successfully determine the bending moments and shear forces of static and modal responses of an isotropic beam with free-free and clamped-free conditions. The reduction in one order of the spatial derivative by using the pulsed speckle shearography system leads to a significant improvement in the computation of bending moments and shear forces. The damage localization based on the analysis of discontinuities and perturbations of the static response produces better results than the one based on the modal response. It was also found that the bending moment indicator is better for the damage localization when it is computed from experimental rotation fields. The reason is that the experimental high frequency noise becomes dominant during the calculation of spatial derivatives. This noise can be partially removed by applying low-pass filters. However, in this process, the higher order signal terms associated to small damages are also removed and, therefore, the damage localization based on disturbances or discontinuities on high order derivatives becomes difficult. 


\section{Acknowledgments}

The authors greatly appreciate the financial support of FCOMP-01-0124-FEDER-010236 through Project Ref. FCT PTDC/EME-PME/102095/2008.

\section{References}

[1] J.V. Araujo dos Santos, N.M.M. Maia, C.M. Mota Soares and C.A. Mota Soares, Structural Damage Identification: A Survey, in: Trends in Computational Structures Technology, chapter 1, Stirlingshire, UK: Saxe-Coburg Publications, 2008, pp. 1-24.

[2] A.K. Pandey, M. Biswas and M.M. Samman, Damage detection from changes in curvature mode shapes, Journal of Sound and Vibration 145(2) (1991), 321-332.

[3] O.S. Salawu and C. Williams, Damage Location Using Vibration Mode Shapes, Proceedings of the 12th International Modal Analysis Conference, Honolulu, Hawai, USA, 1994, pp. 933-939.

[4] N. Stubbs and J.T. Kim, Damage localization in structures without baseline modal parameters, AIAA Journal 34(8) (1996), 1644-1649.

[5] J.T. Kim and N. Stubbs, Crack Detection in Beam-Type Structures Using Frequency Data, Journal of Sound and Vibration 259(1) (2003), $145-160$.

[6] C.P. Ratcliffe and W.J. Bagaria, Vibration technique for locating delamination in a composite beam, AIAA Journal 36(6) (1998), 1074-1077.

[7] J. Maeck and G. De Roeck, Dynamic bending and torsion stiffness derivation from modal curvatures and torsion rates, Journal of Sound and Vibration 225(1) (1999), 153-170.

[8] R.P.C. Sampaio, N.M.M. Maia and J.M.M. Silva, Damage Detection Using the Frequency-Response-Function Curvature Method, Journal of Sound and Vibration 226(5) (1999), 1029-1042.

[9] A. Dutta and S. Talukdar, Damage detection in bridges using accurate modal parameters, Finite Elements in Analysis and Design 40(3) (2004), 287-304.

[10] W. Lestari, P. Qiao and S. Hanagud, Curvature mode shape-based damage assessment of carbon/epoxy composite beams, Journal of Intelligent Material Systems and Structures 18(3) (2007), 189-208.

[11] M. Chandrashekhar and R. Ganguli, Damage assessment of structures with uncertainty by using mode-shape curvatures and fuzzy logic, Journal of Sound and Vibration 326(3-5) (2009), 939-957.

[12] T. Kreis, Handbook of Holographic Interferometry: Optical and Digital Methods, Weinheim, Wiley-VCH, 2005.

[13] J.N. Petzing and J.R. Tyrer, Recent developments and applications in electronic speckle pattern interferometry, The Journal of Strain Analysis for Engineering Design 33(2) (1998), 153-169.

[14] D. Francis, R.P. Tatam and R.M. Groves, Shearography technology and applications: A review, Measurement Science and Technology 21(10) (2010), 102001 (29pp).

[15] H. Glünder and R. Lenz, Fault Detection in Non-Destructive Testing by an Opto-Electronic Hybrid Processor, Proceedings of SPIE 370, 1982, pp. $157-162$.

[16] T. Kreis, W. Jüptner and R. Biedermann, Neural network approach to nondestructive testing, Appl Opt 34(8) (1995), $1407-1415$.

[17] U. Mieth, W. Osten and W. Jüptner, Investigations on the Appearance of Material Faults in Holographic Interferograms, Proceedings of Fringe, 2001, pp. 163-172.

[18] W. Osten, F. Elandaloussi and U. Mieth, Trends for the Solution of Identification Problems in Holographic Non-Destructive Testing (HNDT), Proceedings of SPIE 4900, 2002, pp. 1187-1196.

[19] Y.Y. Hung, Displacement and Strain Measurement, in: Speckle Metrology, R.K. Erf, ed., Academic, New York, 1987 , pp. 51-71.

[20] M. Kalms and W. Osten, Mobile shearography system for the inspection of aircraft and automotive components, Opt Eng 42(5) (2003), 1188-1196.

[21] M.Y.Y. Hung, Y.S. Chen, S.P. Ng, M.S. Shepard, Y. Hou and J.R. Lhota, Review and comparison of shearography and pulsed thermography for adhesive bond evaluation, Opt Eng 46(5) (2007), 051007-1-16.

[22] V.M. Murukeshan, N.C. Keong, V. Krishnakumar, O.L. Seng and A. Asundi, Double shearography for engineering metrology: Optical and digital approach, Optics and Laser Technology 33(5) (2001), 325-328.

[23] F.S. Chau and J. Zhou, Direct measurement of curvature and twist of plates using digital shearography, Optics and Lasers in Engineering 39(4) (2003), 431-440.

[24] B. Bhaduri, M.P. Kothiyal and N.K. Mohan, Curvature measurement using three-aperture digital shearography and fast fourier transform, Optics and Lasers in Engineering 45(10) (2007), 1001-1004.

[25] J.V. Araujo dos Santos, H.M.R. Lopes, M. Vaz, C.M. Mota Soares, C.A. Mota Soares and M.J.M. de Freitas, Damage localization in laminated composite plates using mode shapes measured by pulsed TV holography, Composite Structures 76(3) (2006), $272-281$.

[26] H.M.R. Lopes, R.M. Guedes and M.A. Vaz, An improved mixed numerical-experimental method for stress field calculation, Optics and Laser Technology 39(5) (2007), 1066-1073.

[27] H. Guan and V.M. Karbhari, Improved damage detection method based on element modal strain damage index using sparse measurement, Journal of Sound and Vibration 309(3-5) (2008), 465-494.

[28] H.M.R. Lopes, J.V. Araújo dos Santos, C.M. Mota Soares, R.J. Miranda Guedes and M.A. Pires Vaz, A numerical-experimental method for damage location based on rotation fields spatial differentiation, Computers \& Structures 89(19-20) (2011), 1754-1770.

[29] R.C. Gonzalez and P.A. Wintz, Digital image processing, Reading, Massachusetts, Addison-Wesley, 2nd ed., 1987.

[30] D.C. Ghiglia and M.D. Pritt, Two-Dimensional Phase Unwrapping: Theory, Algorithms, and Software, New York, Wiley, 1998.

[31] G. Pedrini, Y.-L. Zou and H.J. Tiziani, Quantitative Evaluation of Digital Shearing Interferogram Using the Spatial Carrier Method, Pure 
Appl Opt 5(3) (1996), 313-321.

[32] F. Santos, M. Vaz and J. Monteiro, A new set-up for pulsed digital shearography applied to defect detection in composite structures, Optics and Lasers in Engineering 42(2) (2004), 131-140. 

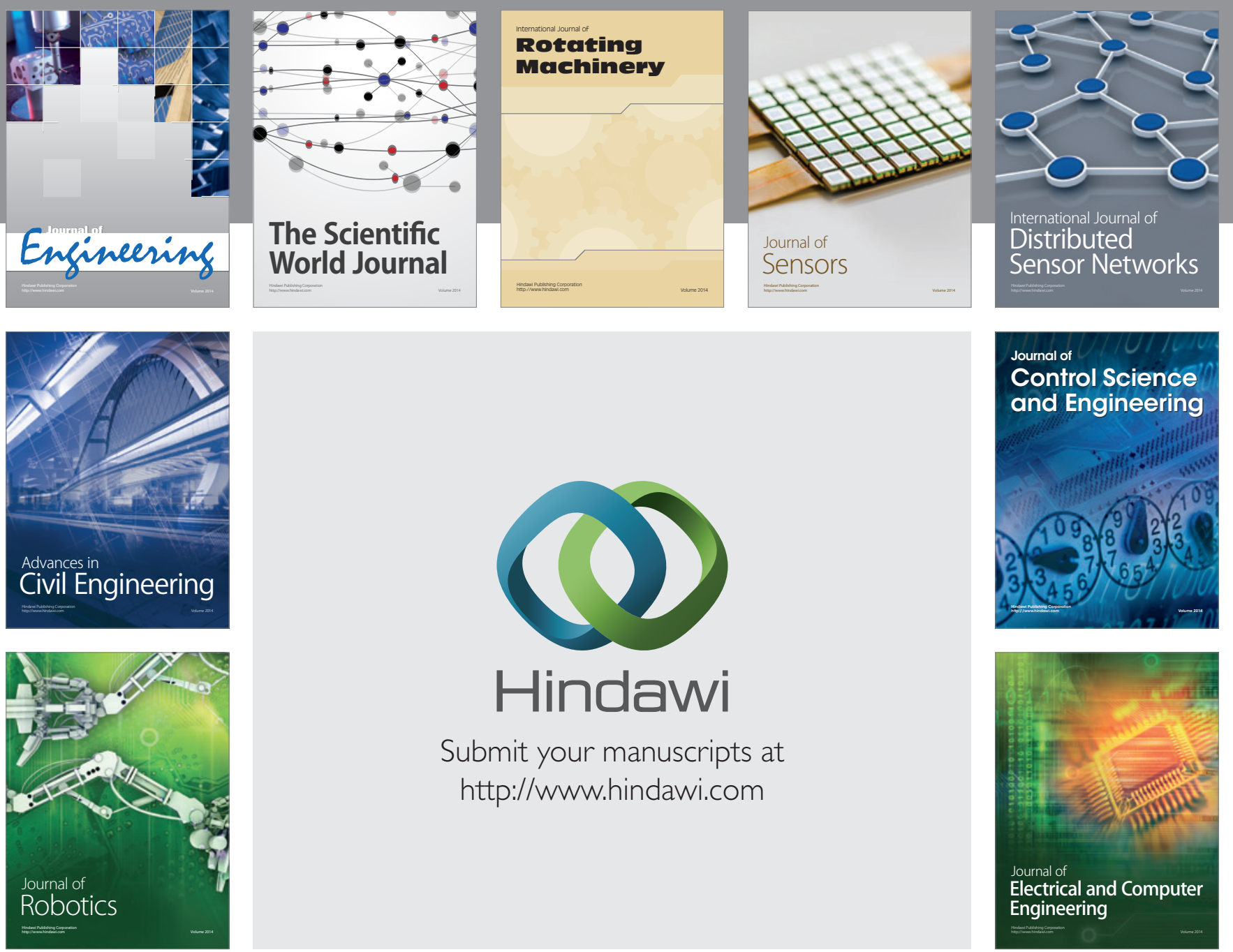

Submit your manuscripts at

http://www.hindawi.com
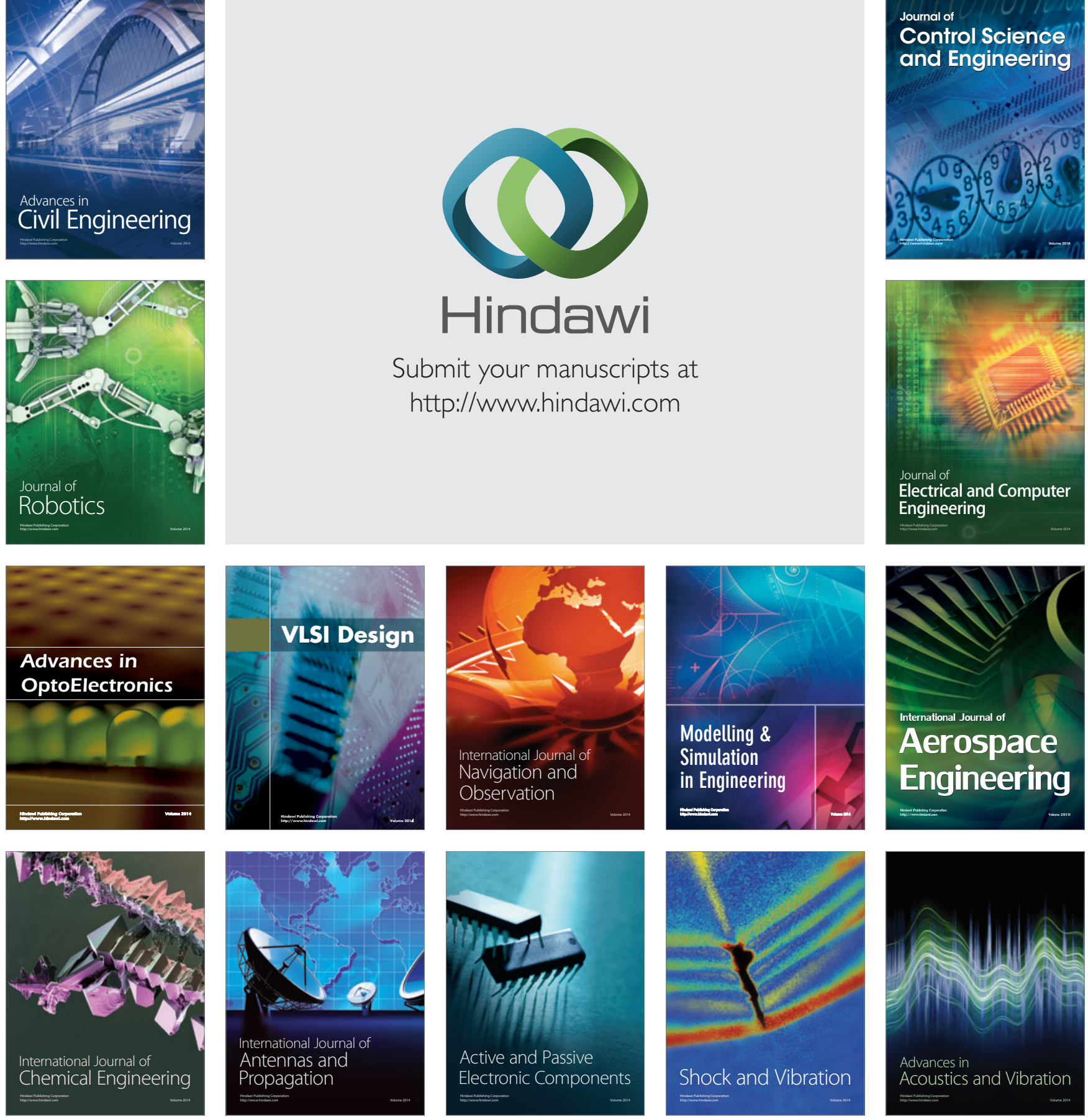